\title{
Ising model on the Apollonian network with node-dependent interactions
}

\author{
R. F. S. Andrade, ${ }^{1,2}$ J. S. Andrade, Jr., ${ }^{2,3}$ and H. J. Herrmann ${ }^{2,3}$ \\ ${ }^{1}$ Instituto de Física, Universidade Federal da Bahia, 40210-210 Salvador, Brazil \\ ${ }^{2}$ Computational Physics, IfB, ETH-Hönggerberg, Schafmattstr. 6, 8093 Zürich, Switzerland \\ ${ }^{3}$ Departamento de Física, Universidade Federal do Ceará, Campus do Pici, 60455-760 Fortaleza, Brazil \\ (Received 23 November 2008; revised manuscript received 7 February 2009; published 16 March 2009)
}

\begin{abstract}
This work considers an Ising model on the Apollonian network, where the exchange constant $J_{i, j}$ $\sim 1 /\left(k_{i} k_{j}\right)^{\mu}$ between two neighboring spins $(i, j)$ is a function of the degree $k$ of both spins. Using the exact geometrical construction rule for the network, the thermodynamical and magnetic properties are evaluated by iterating a system of discrete maps that allows for very precise results in the thermodynamic limit. The results can be compared to the predictions of a general framework for spin models on scale-free networks, where the node distribution $P(k) \sim k^{-\gamma}$, with node-dependent interacting constants. We observe that, by increasing $\mu$, the critical behavior of the model changes from a phase transition at $T=\infty$ for a uniform system $(\mu=0)$ to a $T=0$ phase transition when $\mu=1$ : in the thermodynamic limit, the system shows no true critical behavior at a finite temperature for the whole $\mu \geq 0$ interval. The magnetization and magnetic susceptibility are found to present noncritical scaling properties.
\end{abstract}

DOI: 10.1103/PhysRevE.79.036105

PACS number(s): 89.75.Hc, 05.50.+q, 64.60.aq

\section{INTRODUCTION}

Magnetic models on complex networks have quite distinct behavior from those on regular lattices [1]. Their properties are of far greater importance than just a mathematical curiosity since they establish landmarks for the behavior of many systems, such as social, economic, and communication networks. For such systems, the understanding of the conditions leading to a phase transition, or a sudden collective change in the behavior of the agents, is of utmost importance to avoid a breakdown of social structures or collective current day technological facilities $[2,3]$.

The absence of a finite temperature phase transition in the thermodynamic limit $N \rightarrow \infty$, where $N$ is the number of nodes $[4,5]$, concomitant with the presence of a finite degree of magnetic ordering, stays among the first results that have been obtained for Ising models on the standard BarabasiAlbert (BA) scale-free network [6], where the exponent of the node distribution $P(k) \sim k^{-\gamma}$ assumes the value $\gamma=3$. It was also observed that finite temperature critical behavior is found when $\gamma \in(3,5]$ while, for $\gamma>5$ the critical behavior collapses at $T=0$. Later, an interesting interplay between critical behavior and node-dependent interaction constants has been evidenced [7-9]: if the strength of interactions in a BA network, with a given value $\gamma$, is nonuniformly reduced according to

$$
J_{i, j}=J_{0} /\left(k_{i} k_{j}\right)^{\mu},
$$

where $k_{\ell}$ is the degree of node $\ell$, the critical behavior moves into the universality class of the uniform model with a different value $\gamma^{\prime}$. This makes it possible, for instance, to devise models in the standard BA network that undergo finite temperature phase transition. An analytic expression,

$$
\gamma^{\prime}=(\gamma-\mu) /(1-\mu),
$$

has been derived based on scaling arguments but, although it has been numerically verified for BA networks, it is not known whether its validity extends to other networks.
The purpose of this work is to investigate the effect of a node-dependent coupling constant on the properties of an Ising model on the Apollonian network (AN) [10,11]. The analysis of structures represented by exactly scale invariant graphs, such as ANs, as well as physical models defined on them, takes advantage of invariance properties to derive exact mathematical recursion relations that describe the subject properties in subsequent generations. This provides important insights to the properties of similar structures and models on more natural systems, which share some of the network properties with the AN. Similar structures have also been discussed in the context of small-world hierarchical lattices [12] and pseudofractal scale-free objects [13].

This network has very special features $[14,15]$, including presenting a power-law distribution of node degrees, with exponent $\gamma \simeq 1.585$. Previous results for Ising models on the AN have shown that, for a variety of situations where both ferromagnetic and antiferromagnetic interactions are allowed, phase transition in the thermodynamic limit occur only at $T=\infty[10,16]$. ANs are constructed according to precise geometrical rules, which lead to exact self similar patterns and scaling properties. They are also amenable to mathematical analysis based on renormalization or inflation methods, such as the transfer-matrix (TM) formalism we will use here, which allow for the evaluation of its properties in the thermodynamic limit.

These facts turn this model particularly suited for testing the existence of a finite temperature phase by modulating the coupling constants according to Eq. (1). On the other hand, since the AN geometric rules lead to a well defined value of $\gamma$, there is no general free parameter we can use in the study to verify the validity of Eq. (2). Furthermore, it must be stressed that, despite the fact that AN displays power-law distribution of node degree, it differs substantially from BA network with respect to other topological properties, such as the existence of many closed loops. This is expressed, among other measures, by the clustering coefficient $C$, which is very high $(\sim 0.85)$ for AN and very small $(\sim 1 / N)$ for the BA $[10,11]$. As our results will show later on, the Ising model on 


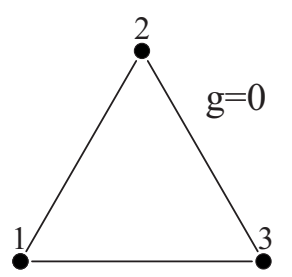

(a)

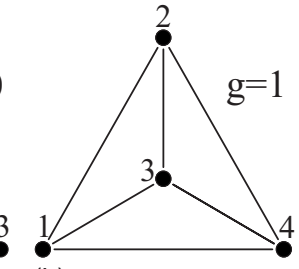

(b)

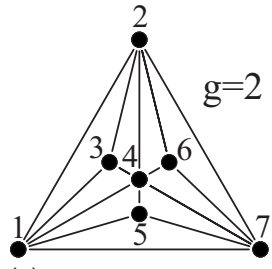

(c)
FIG. 1. Geometrical construction of the first three generations $(g=0,1$, and 2$)$ of the AN. Circles for corresponding Apollonian packing problem are not included. Nodes are numbered according to the scheme used in [16]

AN can hardly be taken into account by the mean-field-like theory that constitutes the main assumption of the evaluation of critical exponents of the Ising model on BA networks [4]. Indeed, it is expected that strong correlations introduced by the abundance of loops in AN modify the classification of critical behavior.

The rest of this paper is organized as follows: Sec. II introduces the basic properties of AN and of the proposed model; details of the used TM scheme to evaluate the thermodynamical properties are discussed in Sec. III. We discuss our main results in Sec. IV, emphasizing the emergence of a crossover in $\mu$. Finally, Sec. V closes the paper with our concluding remarks.

\section{APOLLONIAN NETWORK AND MODEL}

ANs have been recently introduced in the complex network framework $[10,11]$ although the original concepts can be traced back to ancient Greece, where the problem of optimally filling two- and three-dimensional spaces with circles and spheres has been studied by Apollonius of Perga [17]. The complex solution to this problem, which amounts to placing tangent structures with well defined radii at precise centers, suggests the far simpler problem of constructing the AN. In this case, one just has to put a node in each circle center, and a network edge between the centers of each pair of tangent circles. In this work, we consider that, at the zeroth generation $g=0$, three tangent circles with the same radius occupy the centers of an equilateral triangle. Correspondingly, the AN starts with a single equilateral triangle. The construction process can be followed in a recursive way in terms of the generation $g$. For the $(g+1)$ th generation, the network is obtained by inserting a node within each triangle of the $g$ th generation, and connecting it to each of the triangle corners (see Fig. 1). It is a simple matter to verify that the number of network nodes $N(\mathrm{~g})$ and edges $B(\mathrm{~g})$ increase according to, respectively, $N(g)=\left(3^{g}+5\right) / 2$ and $B(g)=\left(3^{g+1}\right.$ $+3) / 2$. The average number of neighbors per node equals six since $B(g) / N(g) \rightarrow 3$ in the limit $g \rightarrow \infty$.

For a given generation $g>0$, the largest node degree is $k_{c}(g)=3 \times 2^{g-1}$, where the subscript $c$ indicates that such node occupies the central network position. The second largest degree nodes, with $k_{e}(g)=2^{g}+1$, occupy the external corners. At any generation $g$, there will be internal nodes with degree $k=k(\bar{g})=3 \times 2^{\bar{g}-1}, \bar{g}=1, \ldots, g$, and $k_{e}$. The number of nodes $m(k ; \bar{g})$ with degree $k(\bar{g})$, or the degree dependent node multiplicity, is expressed by $m(k ; \bar{g})=3^{g-\bar{g}}$ for the internal nodes, and $m\left(k_{e} ; g\right)=3, \forall g$, for the nodes at outer network corners.

As already quoted, the resulting AN is scale-free. However, it also has other properties that are typical for other complex network classes, as being small world (mean minimal path $\langle\ell\rangle \sim \ln N)$, hierarchical [the clustering coefficient of individual nodes $c(k)$ has a power-law dependence on $k$, and having a large clustering coefficient $C$. Because of this, systematic network clustering analysis based on several independent measures [18] shows that AN does not belong to the same class as the most studied network sets, generated by the algorithms proposed by Watts and Strogatz [19] and Barabasi and Albert [6].

We consider the Ising model with spins $\sigma_{i}= \pm 1$ placed on each site of the Apollonian network. Pairs of spins $(i, j)$, which are neighbors on the network, interact with coupling constants $J_{i, j}$. Thus, the Hamiltonian for the system can be written as

$$
H_{g}=-\sum_{(i, j)} J_{i, j} \sigma_{i} \sigma_{j}-h \sum_{i} \sigma_{i}
$$

where $J_{i, j}$ is given by Eq. (1). In our previous studies, we have considered inhomogeneous models, in which the constants $J_{i, j}$ depend on the generation $g$ at which the edge, hence the second spin in the pair, was introduced into the network. Due to the fact that, at each generation, the newly introduced nodes are connected to nodes that were introduced in previous generations, the scheme introduced in Ref. [16] does not assign the values of $J_{i, j}$ according to the rule of Eq. (1). In the following section we discuss how to implement the interaction constants of Eq. (1) in connection with the TM method used to evaluate the model properties.

\section{TM RECURRENCE MAPS}

The basic steps to implement the TM method we used to evaluate the thermodynamic properties have been presented, with some detail, in one of our previous works [16]. However, the method needs to be adapted to the specific situation introduced by the more complex interaction given in Eq. (1). Thus, let us briefly recall that the TM scheme amounts to writing down the partition function $Z[T, h, N(g)]$, where $h$ denotes the magnetic field, for any value of $g$ in terms of a TM that describes the interactions between any two of the outer AN sites. In this process, it is necessary to perform a partial trace over all interaction dependent configurations. Due to the exact geometric AN construction rule, it is possible to express the TM matrix elements at generation $g+1$ in terms of the corresponding elements at generation $g$. In this framework, we basically work with a set of $2 \times 2$ square matrices

$$
M_{g}=\left(\begin{array}{ll}
a_{g} & b_{g} \\
c_{g} & d_{g}
\end{array}\right)
$$

and a set of nonsquare auxiliary matrices 


$$
L_{g}=\left(\begin{array}{cccc}
p_{g} & q_{g} & r_{g} & s_{g} \\
t_{g} & u_{g} & v_{g} & w_{g}
\end{array}\right)
$$

which explicitly include the dependence of the third outer node spin variable. As the $L_{g}$ matrix elements are numbered according to the lexicographic order, the following relations hold: $a_{g}=p_{g}+q_{g}, b_{g}=r_{g}+s_{g}, c_{g}=t_{g}+u_{g}$, and $d_{g}=v_{g}+w_{g}$. For more symmetrical models and field independent situations, the number of independent variables can be reduced.

For several models where the constants $J_{i, j}$ do not depend explicitly on the node degrees, it was possible to write down a single set of recurrence relations between matrix elements in successive generations [16]. Although the basic idea of the method remains the same, for the current model, it is necessary to track the way the nodes are reconnected when they go from $g$ to $g+1$. This influences the change in their degrees so that the same node will contribute differently for distinct values of $g$.

We start the discussion of the changes in the TM scheme by pointing out that, besides knowing the set of node degrees and corresponding degree (node) multiplicity, it is necessary to go one step further and identify each of the $P(g)=\left(g^{2}-g\right.$ $+2) / 2$ different triangles in which the $g$ network can be disassembled. In this respect, each triangle is characterized by the node degrees $k_{i}, k_{j}$, and $k_{\ell}$ of the nodes $i, j$, and $\ell$, respectively. For any triangle and any $g>1$, there is always (only) one node with $k=3$. Note that $P(g)$ grows only with the square of $g$ so that, even for a complex interaction structure, there is practically no constraint to numerically compute these matrix elements for very large values of $g$.

Once this set has been identified, we evaluate the model properties at generation $g$ by computing the contribution to the partition function from each of these $P(g)$ triangles, storing them in corresponding TMs $L_{g}^{i}, i=1, \ldots, P(g)$. To proceed further, we must consider that the $g$ evaluation is equivalent to the $g-1$ one, provided we start with triangular units with a fourth node added at the central position. This way, it is possible to compute the contribution of the new $P(g-1)$ triangles by performing partial trace over the contributions from the central node of each of these structures. The new form of the general recurrence relations for the matrix elements,

$$
\left(L_{g+1}\right)_{i, k}^{\alpha}=\sum_{\ell}\left(L_{g}\right)_{i, j \ell}^{\eta}\left(L_{g}\right)_{i, \ell k}^{\epsilon}\left(L_{g}^{t}\right)_{k, j \ell}^{\delta}
$$

is quite similar to that of the previously investigated models [16]. The difference refers to the superscripts $\eta, \epsilon$, and $\kappa$, which identify which three TMs (corresponding triangles) have been put together. The same arguments can be used again until we obtain one single TM that accounts for the contributions of all network nodes.

The results we present in the next section consider $g$ $\leq 50$ which, for the largest value, the number of sites is roughly of the order of magnitude of the Avogadro number. The adaptation of the uniform TM procedure taking into account the node-dependent interaction constant depends basically in the identification of the basic triangular units and the

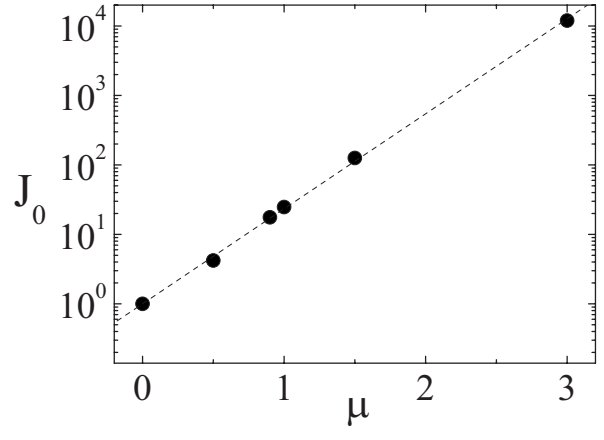

FIG. 2. Dependence of $J_{0}$ on $\mu$, as a result from fixing the ground-state energy per spin $u_{0}=-3$.

assembling rules that combine them when one goes from $g$ to $g+1$. A summary of the implementation of the details is provided in the Appendix.

Finally, it is important to note that the map iterations can be more conveniently performed if we rewrite the set of recurrence maps given by Eq. (6) in terms of the free energy and the ratio of the $L_{g}$ matrix elements to the largest one $\left(q_{g}\right)$. Indeed, this avoids numerical divergences, in the lowtemperature region, when $g$ increases, as conveniently discussed in Ref. [16].

\section{RESULTS}

According to the previous section, we present results for fixed number $g$ of generations, usually $g=10,20,30,40$, and 50 , corresponding to networks with $2.953 \times 10^{4}, 1.743$ $\times 10^{9}, 1.030 \times 10^{14}, 6.079 \times 10^{19}$, and $3.590 \times 10^{23}$ sites, respectively. The precise numerical evaluation of the free energy $f(T, h)$ allows obtaining of the entropy $s(T)$, specific heat $c(T)$, magnetization $m(T, h=0)$, and susceptibility $\chi(T, h=0)$. For models on Euclidian lattice, on hierarchical lattices, and several fractal structures [20], it is possible to show that the correlation length $\xi \sim 1 / \ln \left(\lambda_{1} / \lambda_{2}\right)$, where $\lambda_{1}$ and $\lambda_{2}$ are the two TM eigenvalues. Thus, our framework also allows for the numerical evaluation of $\xi$ with high degree of accuracy. In the case of complex networks and, in particular, of the AN, the connection between $\xi$ and $1 / \ln \left(\lambda_{1} / \lambda_{2}\right)$ is not so obvious, as the distance between two outer nodes remains always one in any generation. Therefore, we will discuss the behavior of $1 / \ln \left(\lambda_{1} / \lambda_{2}\right)$ although we refrain ourselves from calling it $\xi$.

Equation (1) indicates that the coupling constants $J_{i, j}$ linearly depend on $J_{0}$. According to Sec. II, the number of connections in the AN at generation $g$ is $L_{g}=\left(3^{g+1}+3\right) / 2$. If we take $J_{0}=1$ when $\mu=0$, the free energy per spin in the $g$ $\rightarrow \infty$ limit is $u_{0}=-3$ at $T=0$. If we fix $J_{0}=1$ and let $\mu$ increase, the value of $u_{0}$ decreases and, besides that, all thermodynamic effects will occur at a lower value of $T$. Thus, to avoid choosing an adequate temperature scale to work with at each value of $\mu$, we find it more convenient to choose a $\mu$ dependent value $J_{0}(\mu)$, by requiring that $u_{0}=-3, \forall \mu$. In Fig. 2 , we show the dependence of $J_{0}(\mu)$ on $\mu$, which shows that $J_{0} \sim \exp (\mu)$. As a consequence of this choice, all maxima of the specific heat occur roughly at the same value of $T$. 


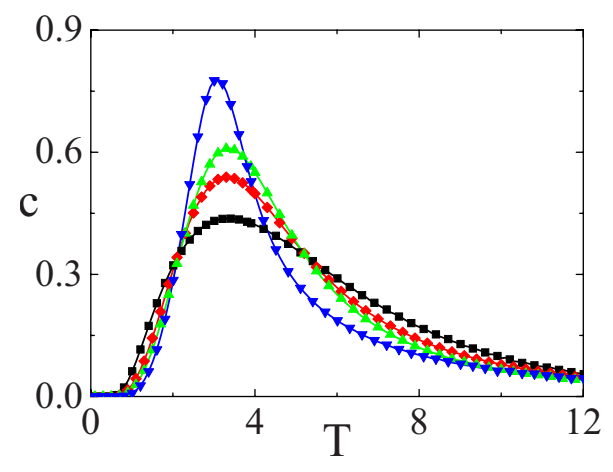

FIG. 3. (Color online) Plots of the specific heat $c$ for $g=40$ (symbols) and $g=50$ (solid line) for different values of $\mu$ : 0 (black squares), 0.5 (red diamonds), 1.0 (green up-triangles), and 3.0 (blue down-triangles). The superposition of curves and symbols for $g=40$ and $g=50$ indicates that $c$ converged to its value in the thermodynamic limit.

Figure 3 shows that, for $g=40$ and 50 , and $\mu=0,0.5,1$, and 3 , the curves for $c$ are qualitatively insensitive to the values of $g$ and $\mu$; all of them are completely smooth, with a Schottky-type maximum at a temperature $T_{s}$. This constitutes a main difference to the results for the $\gamma=3$ BA networks [8]. In that work, the authors report the presence of a finite critical temperature, identified by a jump in the specific heat when $\mu>0.5$, which changes into having a diverging slope when $0.5>\mu>0.33$. According to Eq. (2) and to the AN known value of $\gamma \simeq 1.585$, similar critical behaviors should emerge for $0.854>\mu>0.707$, if the AN were to fall within the BA universality class. This clearly shows that the validity of Eq. (2) cannot, in general, be extended from the BA to other network classes, even if they are scale-free as the AN.

The same calculations reveal that the resulting patterns for $m, \chi$, and $1 / \ln \left(\lambda_{1} / \lambda_{2}\right)$ depend, first, on the generation $g$, and further on whether $0<\mu<1, \mu=1$, and $\mu>1$. So it is adequate to discuss them separately.

\section{A. $0<\boldsymbol{\mu}<\mathbf{1}$}

Within this parameter interval, we observe that, like for the models where $J_{i, j}$ does not depend on node degree, $1 / \ln \left(\lambda_{1} / \lambda_{2}\right)$ numerically diverges $\left(>10^{100}\right)$ for a nonzero temperature $T_{d}(g)$, which increases linearly with $g$. Since $g$ $\sim \ln N, T_{d}$ depends in a logarithmic way on the system size. If we write $T_{d}(g) \sim A(\mu) \ln N$, we find that $A(\mu)$ decreases with $\mu$ (see Fig. 4).

In Fig. 5, the behavior of the zero-field magnetization $m[T, h=0, N(g)]$, which is exactly one when $T=0$, slowly decreases when $T$ increases. Its behavior when $\mu=0.5$, at larger values of $T$, is a bit more complex than that for $\mu=0$ [Fig. 5(a)]. There it is clear that $m$ suffers a first crossover to an exponential decay at $T_{s}$, which is followed by a transition to a second exponential decay, mediated by a larger constant, at $T_{m}(g)$. The magnetization curves for different $g$ collapse during the first and second regimes. The third regime will later on be interrupted again by a smoother decay. As observed with $T_{d}(g), T_{m}(g) \sim B(\mu) g$, with $B(\mu) \sim A(\mu)$. However, $T_{d}$ and $T_{m}$ do not coincide. The second part of the

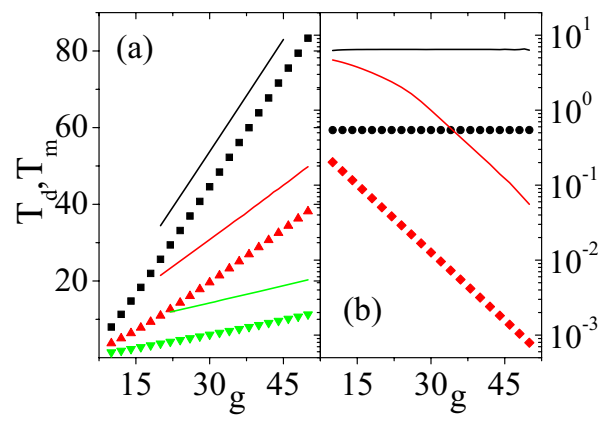

FIG. 4. (Color online) Dependence of the temperatures $T_{d}$ (symbols) and $T_{m}$ (solid lines) on the network generation $g$. They indicate, respectively, the temperature of numerical divergence of $1 / \ln \left(\lambda_{1} / \lambda_{2}\right)$ and of the maximum of susceptibility $\chi$. (a) $\mu=0.0$ (squares), 0.5 (up-triangles), and 0.8 (down-triangles). (b) $\mu=1.0$ (circles) and 1.2 (diamonds).

magnetization curves, where $m[T, 0, N(g)]$ overlaps for different values of $g$, extends over wider $T$ intervals when $g$ increases. This shows that, in the thermodynamical limit $g$ $\rightarrow \infty$, the value of $m$ will follow the second exponential decay when $T \rightarrow \infty$. Nevertheless, as observed for $T_{d}$, this region grows logarithmically with the network size.

The behavior of $\chi$ is strongly correlated with that of $m$. It vanishes when $T \rightarrow 0$, then it grows with $T$, shows a first maximum at a $g$ independent $T_{s}$, and a second $g$ dependent maximum at $T_{m}$. As for $m$, the curves for larger values of $g$ overlap for much larger distances. The maxima of the $\chi$ curves are described by a universal function, as can be ob-
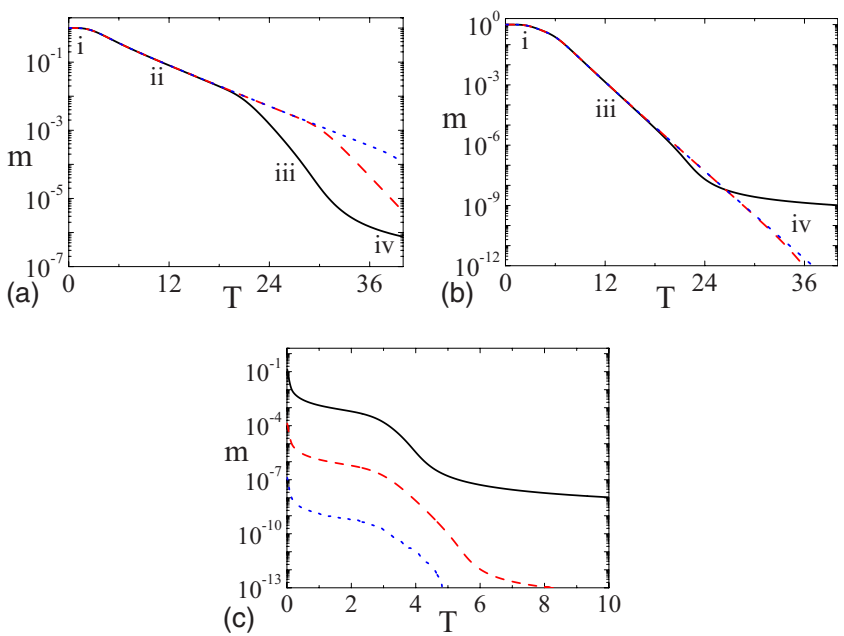

FIG. 5. (Color online) Behavior of the magnetization $m[T, h=0 ; N(g)]$ as function of the temperature $T$ for different values of $g$ when $\mu=0.5,1.0$, and 1.5. (a) $\mu=0.5, g=20,30$, and 40 indicated by dots, dashes, and solid lines. Four regions characterized by different behaviors are obtained: two of them are $g$ independent, the second of which has an exponential decay. The third region starts with a crossover to a second exponential regime. (b) $\mu=1.0$, same symbols as in (a). The first exponential region has disappeared. (c) $\mu=1.5, g=10,15$, and 20 indicated by dots, dashes, and solid lines. The first region in (a) has disappeared. Two $T$ intervals separated by a $g$ dependent crossover temperature are observed. As $g$ increases, $m$ vanishes for any $T>0$. 

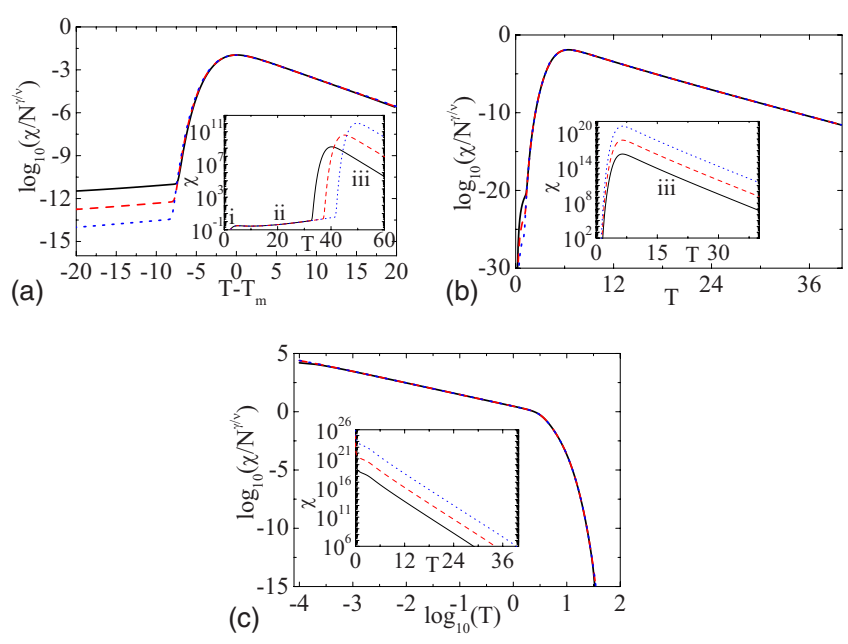

FIG. 6. (Color online) Main panels show scaling properties of the magnetic susceptibility $\chi[T, h=0 ; N(g)]$ as function of the temperature $T$ for different values of $g=40$ (solid), 45 (dashes), and 50 (dots), when (a) $\mu=0.5$, (b) 1.0, and (c) 1.5. The insets show the same curves in the original variables. (a) Scaling properties of $\chi$ as function of $T-T_{m}$, with exponent $\gamma / \nu=0.607$, are observed in region iii of the magnetization $m$ [see Fig. 5(a)]. (b) Scaling properties of $\chi$ as function of $T$, with exponent $\gamma / \nu=1.0$, valid in region iii, with exponential decrease in $m$, as discussed in Fig. 5(b). (c) Scaling properties of $\chi$ as function of $T$, with exponent $\gamma / \nu=1.0$. Scaling is valid in the two regions shown in Fig. 5(c). The horizontal axis is in logarithmic scale to evidence Curie's law with $g$ dependent constant $\mathcal{C}_{g}$.

served in the very precise rescaled curves in Fig. 6(a), which shows that the scaling exponents increase with $\mu$. Note that only the value of $\chi$ needs to be scaled by the corresponding maxima while the location at the temperature axis is corrected by shifting the scale by $T_{m}(g)$. The linear increase in the value of $T_{m}$ as function of $g$ excludes any possibility of having a critical phenomenon associated with susceptibility maxima.

\section{B. $\mu=1$}

This value of $\mu$ determines a crossover in the behavior of the system, which is reflected both in $m$ and $\chi$. This change can be noticed in Fig. 3, which shows that $A(\mu=1)=B(\mu$ $=1)=0$, i.e., the temperatures associated with the maxima of the susceptibility and the divergence of $1 / \ln \left(\lambda_{1} / \lambda_{2}\right)$ become independent of the system size. The precise value of $T_{d}$ depends, of course, on the threshold value of the numerical divergence. However, by plotting the value of $1 / \ln \left(\lambda_{1} / \lambda_{2}\right)$ as function of $1 / T$, we notice a linear dependence in the $T$ $\rightarrow 0$ limit, suggesting that $T_{d}=0$.

However, this new behavior cannot be associated with the emergence of criticality. First we recall that Fig. 3 does not indicate any change in the Schottky profile and, second, we see that $T_{m}>T_{s}$. Finally, the $\chi$ curves in the region around $T_{m}$, which shows a perfect scaling with respect to $g$ with scaling exponent 1, are completely smooth [see Fig. 6(b)]. Note that the horizontal axis indicates that it is not necessary to shift temperature as in Fig. 6(a). Note that the two maxima, which were observed when $\mu<1$, have merged together, and that the large temperature side is characterized by an exponential decay.

As for the previous $\mu$ interval, the behavior of $m$ is strongly correlated with that for $\chi$. It is characterized by a single exponential decay after $T_{m}$, with a very large constant, as shown in Fig. 5(b).

\section{C. $\boldsymbol{\mu}>\mathbf{1}$}

In the last range of parameter values, $T_{d}$ and $T_{m}$ decrease with respect to $g$. As shown by Fig. 4(c), both values converge exponentially to zero with respect to $g$. Figure 4(c) also shows that the rate in the exponential increases with $\mu$.

Therefore, the behavior of $1 / \ln \left(\lambda_{1} / \lambda_{2}\right)$ is different from that one observed for $\mu=1$, the divergence when $T \rightarrow 0$ becomes slower at increasing values of $g$. This suggests that, when $g \rightarrow \infty$, any collective spin ordering is weaker than that of an Ising chain, rather typical for a paramagnetic situation.

The shape of the $m$ curves becomes completely different. The stable plateau at $m=1$ for a finite temperature interval, which survived until $\mu=1$, disappears as $g$ increases, indicating that no spontaneous magnetization exists for a $T>0$. This suggests that, in the thermodynamic limit, $m \equiv 0 \forall T$ $>0$. Our results do not allow to assert that $m \equiv 0$ also for $T=0$.

The behavior of $\chi$ supports the conclusion of a lowtemperature paramagnetic phase. It increases very rapidly from zero to a maximum value at $T_{m}$, followed by a $1 / T$ law. Since $T_{m}$ goes exponentially to zero, a Curie law prevails for large $g$. This is shown by the scaled curves in Fig. 6(c), which indicate that the Curie constant $\mathcal{C}$ depends on $g$.

\section{DISCUSSION AND CONCLUSIONS}

The results we obtained for the magnetic behavior of the Ising model with node-dependent interaction constants reveal a quite rich picture although no critical behavior at a finite temperature has been identified. The properties of specific heat show that the $g$ dependent curves converge very rapidly to a well defined value in the thermodynamic limit. On the other hand, magnetization and susceptibility indicate a much more complex behavior which, for certain temperature intervals, are heavily dependent on the value of $g$.

The TM method allows for the comparison of $m$ and $\chi$ for different values of $g$, which leads to the identification that part of the results are due to finite-size events. The curves showing such effects are amenable to very precise collapsing by adequate scaling expressions, similar to critical points in magnetic models on Euclidian lattices. This includes the dependence of characteristic values of the temperature $\left(T_{d}\right.$ and $\left.T_{m}\right)$.

The behavior of the system in the region $\mu<1$ is close to that observed for magnetic system with uniform interactions on BA networks: only an ordered phase is observed at any value of $T . \mu=1$ characterizes a crossover in the behavior of the system; as for $\mu<1$ the magnetization vanishes, for any value of $T$, when $g \rightarrow \infty$. This region reveals a typical behavior of a genuine paramagnetic system. This picture is cor- 
roborated by the behavior of $\chi$, as one finds that a Curie law is valid in a limited region close to $T=0$. For larger values of $T$, the decay is characterized by an exponential decay.

In the context of complex networks our most important finding is that the relation of Eq. (1) between effective topology and interaction strength proposed in Refs. [7,8] does not have general validity for all scale-free networks since the Apollonian case behaves differently.

The developed framework can be adapted to investigate the Ising model (or other magnetic models) with nodedependent interactions on similar networks. The expressions indicated in the Appendix are valid also for antiferromagnetic couplings $J_{0}<0$ and/or for negative values of $\mu$ in the AN. For this particular situation $J_{0}>0, \mu<0$, preliminary results indicate that the thermodynamical behavior is much the same as long as $|\mu|$ is small. However, for larger values of $|\mu|$, Eq. (1) reduces significantly the interaction between nodes with low degree, which are the majority. Therefore, a decrease in magnetic ordering may, in the limit $N \rightarrow \infty$, be expected. A thorough analysis of the unexplored parameter regions will be presented in a forthcoming paper.

\section{ACKNOWLEDGMENT}

R.F.S.A. and J.S.A., Jr. thank CNPq for financial support.

\section{APPENDIX}

As discussed in the Sec. II, for any generation $g$, the node occupying the central position of the AN has the largest degree $k_{c}(g)$, the value of which results from the difference equation relating the values of $k_{c}$ at two successive generations: $k_{c}(g+1)=2 k_{c}(g)$. The degree of the nodes at the external corners obeys a similar equation, namely: $k_{e}(g+1)$ $=2 k_{e}(g)-1$.

The AN can be disassembled in triangles, in such a way that each node $i$ of degree $k_{i}$ belongs to $k_{i}$ triangles. The only exception refers to the nodes at the external corners, which have degree $k_{e}(g)$ but belong to $k_{e}(g)-1$ triangles. Each such triangle can be characterized by the degree of its three nodes. The number $P(g)$ of different triangles at generation $g$ can be expressed in terms of $\rho(g)$ and $\tau(g)$, respectively, the number of triangles that does not include (includes) an external node: $P(g)=\rho(g)+\tau(g)$. Since they obey the relations $\rho(g+1)$ $=\rho(g)+\tau(g)-1$ and $\tau(g+1)=\tau(g)+1$, we obtain $\rho(g)=\left(g^{2}\right.$ $-3 g+2) / 2$ and $\tau(g)=g$, from which the expression for $P(g)$ anticipated in Sec. III follows. The number $\rho(g)$ can be further decomposed in terms of $\iota(g)$ and $\kappa(g)$, respectively, the number of different triangles that includes (does not include) the central node at generation $g$. It is a simple matter of inspection to see that $\iota(g)=g-2$ and $\kappa(g)=\left(g^{2}-5 g+6\right) / 2$, $\forall g \geq 2$.

For the purpose of computing the TMs, it is necessary to identify the distinct triangles present in the AN. This proceeds by the collection $T_{g, \zeta}\left(k_{1}, k_{2}, k_{3}\right)$, where $g$ indicates the generation, $\zeta$ is a number $\in[1, P(g)]$, and $k_{i}$ indicate the degrees of the nodes at the vertices of the triangle. $T_{g, \zeta}\left(k_{1}, k_{2}, k_{3}\right)$ are recursively defined according to the following rules:
(1) $k_{3}=3, \forall g, \forall \zeta$.

(2) Since $\tau(g)=g$, one single new triangle containing an external node is introduced into the network, $\forall g$. We use $\zeta$ $=P(g-1)+1$ to characterize it, and note further that $k_{1}$ $=k_{e}(g), k_{2}=k_{c}(g)$. Any such triangle retains the $k_{i}$ values for all further generations, i.e.,

$$
T_{g, \zeta}\left(k_{1}, k_{2}, k_{3}\right)=T_{g, \zeta^{\prime}}\left(k_{1}, k_{2}, k_{3}\right), \forall \zeta^{\prime} \geq \zeta .
$$

(3) $\forall g$, there are $\rho(g)$ different triangles, among which $\kappa(g)$ have been introduced in previous generations. They will be characterized by the same values of $k_{i}$ so that Eq. (A1) also holds for this subset. The remaining $\iota(g)$ new triangles are numbered according to the rule: $\zeta=P(g-1)+\ell$, $\ell=2,3, \ldots, g-1$. For each value of $\ell$, we set $k_{1}=k_{c}(g), k_{2}$ $=k_{c}(\ell)$.

The final step consists of establishing the rule to combine the contributions to the partition function from three distinct triangles at generation $g$ to obtain the partition function at generation $g+1$ according to Eq. (6). If the properties of the systems are to be computed until a chosen value $g$, we are required to start with $P(g)$ distinct triangles, precisely identified as discussed above. Then, as discussed in Sec. III, it is necessary to define a map that selects the proper values of $\eta, \epsilon, \delta \in[1, P(g)]$ used to perform the trace over the common central node of the triangle $\alpha \in[1, P(g-1)]$. So let us note that

$$
[1, P(g)]=[1] \cup\left\{\bigcup_{j=2}^{g}[P(g-1)+1, P(g-1)+j-1]\right\} .
$$

Then, the values of $\eta, \epsilon$, and $\delta$ are given, as function of $\alpha$, by the following expressions:

$$
\begin{gathered}
\alpha=1: \\
\eta=1, \epsilon=\delta=2 ; \\
\alpha=P(j-1)+1, j \in[2, g-1]: \\
\eta=2, \epsilon=P(j)+1, \delta=P(j)+2 ; \\
\eta \in[P(j-1)+2, P(j-1)+j-1], j \in[2, g-1]: \\
\eta[\alpha-P(j-1)]+2, \epsilon=P(j)+2, \delta=\alpha+j .
\end{gathered}
$$

As the AN is self-similar, these maps are also valid for all forthcoming partial trace operations until only one single triangle is left. At this step, the remaining TM contains the contribution from all spin configurations, from which the thermodynamical properties follow.

With the help of these relations, a set of recurrence maps can derived from Eq. (5), which allows for the evaluation of the free energy and its derivatives:

$$
\begin{aligned}
f_{g+1}^{\alpha}= & \frac{N_{g}}{N_{g+1}}\left(f_{g}^{\eta}+f_{g}^{\epsilon}+f_{g}^{\delta}\right)-\frac{T}{N_{g+1}}\left\{\operatorname { l n } \left[1+x_{1, g}^{\eta} x_{1, g}^{\epsilon} x_{1, g}^{\delta}\right.\right. \\
& \times \exp (-2 \beta h)]\}-\frac{h}{N_{g+1}},
\end{aligned}
$$




$$
\begin{gathered}
x_{1, g+1}^{\alpha}=\frac{x_{2, g}^{\epsilon} x_{2, g}^{\delta}+x_{1, g}^{\eta} x_{3, g}^{\epsilon} x_{3, g}^{\delta} \exp (-2 \beta h)}{1+x_{1, g}^{\eta} x_{1, g}^{\epsilon} x_{1, g}^{\delta} \exp (-2 \beta h)}, \\
x_{2, g+1}^{\alpha}=\frac{x_{2, g}^{\eta} x_{4, g}^{\delta}+x_{3, g}^{\eta} x_{1, g}^{\epsilon} x_{2, g}^{\delta} \exp (-2 \beta h)}{1+x_{1, g}^{\eta} x_{1, g}^{\epsilon} x_{1, g}^{\delta} \exp (-2 \beta h)}, \\
x_{3, g+1}^{\alpha}=\frac{x_{2, g}^{\eta} x_{2, g}^{\epsilon} x_{6, g}^{\delta}+x_{3, g}^{\eta} x_{3, g}^{\epsilon} x_{7, g}^{\delta} \exp (-2 \beta h)}{1+x_{1, g}^{\eta} x_{1, g}^{\epsilon} x_{1, g}^{\delta} \exp (-2 \beta h)}, \\
x_{4, g+1}^{\alpha}=\frac{x_{4, g}^{\eta} x_{4, g}^{\varepsilon}+x_{5, g}^{\eta} x_{5, g}^{\varepsilon} x_{1, g}^{\delta} \exp (-2 \beta h)}{1+x_{1, g}^{\eta} x_{1, g}^{\varepsilon} x_{1, g}^{\delta} \exp (-2 \beta h)}, \\
x_{5, g+1}^{\alpha}=\frac{x_{4, g}^{\eta} x_{6, g}^{\epsilon} x_{2, g}^{\delta}+x_{5, g}^{\eta} x_{7, g}^{\epsilon} x_{3, g}^{\delta} \exp (-2 \beta h)}{1+x_{1, g}^{\eta} x_{1, g}^{\epsilon} x_{1, g}^{\delta} \exp (-2 \beta h)},
\end{gathered}
$$

$$
\begin{aligned}
& x_{6, g+1}^{\alpha}=\frac{x_{6, g}^{\eta} \epsilon_{4, g}^{\epsilon} x_{4, g}^{\delta}+x_{7, g}^{\eta} x_{5, g}^{\epsilon} x_{5, g}^{\delta} \exp (-2 \beta h)}{1+x_{1, g}^{\eta} x_{1, g}^{\epsilon} x_{1, g}^{\delta} \exp (-2 \beta h)}, \\
& x_{7, g+1}^{\alpha}=\frac{x_{6, g}^{\eta} x_{6, g}^{\epsilon} x_{6, g}^{\delta}+x_{7, g}^{\eta} x_{7, g}^{\epsilon} x_{7, g}^{\delta} \exp (-2 \beta h)}{1+x_{1, g}^{\eta} x_{1, g}^{\epsilon} x_{1, g}^{\delta} \exp (-2 \beta h)} .
\end{aligned}
$$

In the above relations, the following variables have been used:

$$
\begin{gathered}
x_{1, g}=\frac{q_{g}}{p_{g}}, \quad x_{2, g}=\frac{r_{g}}{p_{g}}, \quad x_{3, g}=\frac{s_{g}}{p_{g}}, \\
x_{4, g}=\frac{t_{g}}{p_{g}}, \quad x_{5, g}=\frac{u_{g}}{p_{g}}, \quad x_{6, g}=\frac{v_{g}}{p_{g}}, \\
x_{7, g}=\frac{w_{g}}{p_{g}} .
\end{gathered}
$$

[1] S. N. Dorogovtsev, A. V. Goltsev, and J. F. F. Mendes, Rev. Mod. Phys. 80, 1275 (2008).

[2] L. F. Costa, F. A. Rodrigues, G. Travieso, and P. R. Villas Boas, Adv. Phys. 56, 167 (2007).

[3] S. Boccaletti, V. Latora, Y. Moreno, M. Chavez, and D.-U. Hwang, Phys. Rep. 424, 175 (2006).

[4] S. N. Dorogovtsev, A. V. Goltsev, and J. F. F. Mendes, Phys. Rev. E 66, 016104 (2002).

[5] A. Aleksiejuk, J. A. Holyst, and D. Stauffer, Physica A 310, 260 (2002).

[6] A.-L. Barabási and R. Albert, Science 286, 509 (1999).

[7] J. O. Indekeu, Physica A 333, 461 (2004).

[8] C. V. Giuraniuc, J. P. L. Hatchett, J. O. Indekeu, M. Leone, I. Perez Castillo, B. Van Schaeybroeck, and C. Vanderzande, Phys. Rev. Lett. 95, 098701 (2005).

[9] C. V. Giuraniuc, J. P. L. Hatchett, J. O. Indekeu, M. Leone, I. Perez Castillo, B. Van Schaeybroeck, and C. Vanderzande, Phys. Rev. E 74, 036108 (2006).

[10] J. S. Andrade, Jr., H. J. Herrmann, R. F. S. Andrade, and L. R. daSilva, Phys. Rev. Lett. 94, 018702 (2005).
[11] J. P. K. Doye and C. P. Massen, Phys. Rev. E 71, 016128 (2005).

[12] M. Hinczewski and A. N. Berker, Phys. Rev. E 73, 066126 (2006).

[13] S. N. Dorogovtsev, A. V. Goltsev, and J. F. F. Mendes, Phys. Rev. E 65, 066122 (2002).

[14] D. J. B. Soares, J. S. Andrade, H. J. Herrmann, and L. R. daSilva, Int. J. Mod. Phys. C 17, 1219 (2006).

[15] A. A. Moreira, D. R. Paula, R. N. Costa Filho, and J. S. Andrade, Phys. Rev. E 73, 065101(R) (2006).

[16] R. F. S. Andrade and H. J. Herrmann, Phys. Rev. E 71, 056131 (2005).

[17] H. J. Herrmann, G. Mantica, and D. Bessis, Phys. Rev. Lett. 65, 3223 (1990).

[18] L. da Fontoura Costa and R. F. S. Andrade, New J. Phys. 9, 311 (2007).

[19] D. J. Watts and S. H. Strogatz, Nature (London) 393, 440 (1998).

[20] R. F. S. Andrade, Phys. Rev. E 61, 7196 (2000). 